\title{
Discrete Vortex Representation of Magnetohydrodynamics
}

\author{
R. Kinney, T. Tajima, N. Petviashvili \\ Institute for Fusion Studies \\ The University of Texas at Austin \\ Austin, Texas 78712 \\ and \\ J.C. McWilliams \\ National Center for Atmospheric Research \\ P.O. Box 3000 \\ Boulder, Colorado 80307
}

DOE/ET/53088--592

DE93 012190

October 25, 1992

\begin{abstract}
We present an alternative approach to statistical analysis of an intermittent ideal MHD fluid in two dimensions, based on the hydrodynamical discrete vortex model applied to the Elsasser variables. The model contains negative temperature states which predict the formation of magnetic islands, but also includes a natural limit under which the equilibrium states revert to the familiar twin-vortex states predicted by hydrodynamical turbulence theories. Numerical dynamical calculations yield equilibrium spectra in agreement with the theoretical predictions.
\end{abstract}


Given a discrete representation of the continuous equations for an inviscid fluid, a statistical theory can be constructed, and, even though such systems are not in thermodynamic equilibrium at the molecular level, one can expect real systems to tend towards the statistically favored states during time scales for which the model is valid. For numerical simulation, of course, some discretization of the continuous system is also necessary.

Two methods of discretizing hydrodynamical turbulence have been employec: a decomposition of field variables into Fourier modes, and a representation of the fluid as a collection of discrete vortices. A statistical Fourier analysis for two-dimensioral magnetohydrodynamics (MHD) has already been undertaken [1]. In this letter, we show that a point-vortex discretization like that of hydrodynamics (or the equivalent guiding-center plasma [2]) is also possible for 2-D MHD, and we give results based on statistical theory as well as direct numerical simulation of the vortex system.

Why is such an approach worth taking? It has been asserted [3] that different approaches to discretization of functional integrations cannot in general be expected to yield equivalent results. Indeed, in the hydrodynamical studies, the two different discretization approaches, while both making similar qualitative predictions about a cascade of energy to low wave-numbers, do not yield the same results [4]. In a neutral fluid, it is known that small dissipation causes the fluid to form intermediate-scale vorticity distributions, sometimes called coherent structures [5]. Some statistical theories of neutral fluids are emerging which account for these structures, which dominate the non-linear evolution $[6,7]$. Meanwhile, a great amount of literature has been written on the dynamics of point vortices in 2-D fluids (Refs. $[8,9,10,11,12]$ and many others), and such models have been used to predict end-states of the flow [i3], and the fluid's dynamics evolution towards that state [14]. These successes are encouraging and make the prospect of analogous phenomena in 2-D MHD exciting. Computationally, discrete-vortex models can qualitatively reproduce the behavior of the primitive fluid equations at a lower cost than a spectral or grid point code [15]. In 
addition, there is the possibility of modeling more general large-scale filamentary structures $[16,17]$. Taking an analogous approach to MHD simulations could lead to similarly efficient numerical models of magnetic turbulence.

There is mounting observational evidence of intermittent plasma structures occurring in high- $\beta$ astrophysical plasmas [18]. In a high- $\beta$ plasma, in which the fluid pressure dominates the magnetic pressure, it seems reasonable to expect that the emergence of coherent structures in both vorticity and current is a natural phenomenon. Because laboratory fusion plasmas are run with very low $\beta$, numerical simulations give the best opportunity to search for high- 3 intermittency under controlled conditions. Recent high-resolution MHD simulations have in fact displayed strongly intermittent states in 2-D plasmas [19], which adds to our confidence that a treatment of plasma turbulence based on coherent structures will prove to be appropriate.

Long ago, Elsasser [20] pointed out that the basic equations of ideal MHD can be written in the form

$$
\begin{gathered}
\frac{\partial \boldsymbol{u}}{\partial t}+(\boldsymbol{w} \cdot \nabla) \boldsymbol{u}=-\nabla \eta ; \boldsymbol{u}=\boldsymbol{v}+\boldsymbol{B} \\
\frac{\partial \boldsymbol{w}}{\partial t}+(\boldsymbol{u} \cdot \nabla) \boldsymbol{w}=-\nabla \eta ; \boldsymbol{w}=\boldsymbol{v}-\boldsymbol{B} \\
\boldsymbol{\nabla} \cdot \boldsymbol{u}=\boldsymbol{\nabla} \cdot \boldsymbol{w}=0, ; \eta=\frac{p}{\rho}+\boldsymbol{B}^{2} .
\end{gathered}
$$

Velocities are measured in units of an arbitrary constant $v_{0}$, and the magnetic field is measured in units of $B_{0}=\sqrt{4 \pi \rho v_{0}^{2}}$.

Let us also define functions $\Omega$ and $\boldsymbol{A}$ by

$$
\begin{gathered}
\Omega^{u}=\boldsymbol{\nabla} \times \boldsymbol{u} ; \boldsymbol{u}=\boldsymbol{\nabla} \times \boldsymbol{A}^{u} \\
\Omega^{w}=\boldsymbol{\nabla} \times \boldsymbol{w} ; \boldsymbol{w}=\boldsymbol{\nabla} \times \boldsymbol{A}^{w} .
\end{gathered}
$$

We will either use a general species superscript to indicate $u$ or $w$ or omit any superscript 
to generically indicate both possibilities.

In a neutral fluid, the vorticity is conservatively advected through the fluid. We seek an analogous result for our $\Omega$ 's. Defining the total advective derivative opcrators

$$
D^{u} \boldsymbol{V} \equiv \boldsymbol{\nabla} \times(\boldsymbol{u} \times \boldsymbol{V}), D^{w} \boldsymbol{V} \equiv \boldsymbol{\nabla} \times(\boldsymbol{w} \times \boldsymbol{V})
$$

one finds that the curl of the momentum equation becomes

$$
D^{u} \Omega^{w}+D^{w} \Omega^{u}=0
$$

while the induction equation may be written

$$
D^{w} \Omega^{u}-D^{u} \Omega^{w}=\boldsymbol{S}
$$

in which there is a source term

$$
\boldsymbol{S}=2 \boldsymbol{\Omega}^{w} \times \boldsymbol{\Omega}^{u}+2\left[\boldsymbol{\Omega}^{w} \cdot \nabla \boldsymbol{u}-\boldsymbol{\Omega}^{u} \cdot \nabla \boldsymbol{w}\right]+\nabla^{2}(\boldsymbol{u} \times \boldsymbol{w})-\left(\nabla^{2} \boldsymbol{u}\right) \times \boldsymbol{w}-\boldsymbol{u} \times\left(\nabla^{2} \boldsymbol{w}\right)
$$

In two dimensions, $\boldsymbol{\Omega}=\Omega \hat{\boldsymbol{z}}$ and $\boldsymbol{A}=A \hat{\boldsymbol{z}}$, and most of the terms in $\boldsymbol{S}$ vanish identically. What remains can be written

$$
\boldsymbol{S}=S^{\prime} \hat{\boldsymbol{z}}=\left[\partial_{i} A^{u}, \partial_{i} A^{w}\right] \hat{\boldsymbol{z}}
$$

where $[\cdots]$ indicates the Poisson bracket in two dimensions.

Let our plasma be highly intermittent in its spatial distribution, consisting of a number of $u$ and $w$ filaments with positions $\left\{\boldsymbol{x}_{\boldsymbol{i}}\right\}$ and intensities $\left\{\alpha_{i}\right\}$. The $\Omega$ 's then take the form

$$
\Omega=\sum_{i} \alpha_{i} \delta\left(\boldsymbol{x}-\boldsymbol{x}_{i}\right) .
$$

The variables $\left\{\boldsymbol{x}_{i}\right\}$ and $\left\{\alpha_{i}\right\}$ fully determine the fields $\boldsymbol{u}, \boldsymbol{w}$, and $S$ through eqs.(8), (2), and (7). Equations(4) and (5) are solved by the motion of the filaments if

$$
\begin{aligned}
\frac{d \boldsymbol{x}_{i}^{u}}{d t} & =\boldsymbol{w}\left(\boldsymbol{x}_{i}^{u}\right), \frac{d \alpha_{i}^{u}}{d t}=S\left(\boldsymbol{x}_{i}^{u}\right), \\
\frac{d \boldsymbol{x}_{i}^{\prime \nu}}{d t} & =\boldsymbol{u}\left(\boldsymbol{x}_{i}^{w}\right), \frac{d \alpha_{i}^{w}}{d t}=-S\left(\boldsymbol{x}_{i}^{w}\right) .
\end{aligned}
$$


The induction of current represented by the source term is manifested by a simultaneous increase in the strength of $u$-filaments and a decrease in the strength of $w$-filaments. The fact that $u$ and $w$ filaments are not necessarily coincident and that filaments are not guaranteed to be present wherever $S$ is non-zero makes this discretization approximate, but acceptable for large number densities of filaments.

Of importance in $\mathrm{E}_{4} .(5)$ is the fact that $\boldsymbol{S}$ contains no derivatives of $\boldsymbol{\Omega}$, whereas $D \boldsymbol{\Omega}$ contains first-order derivatives of $\Omega$ in both space and time. Thus, if we are interested in tracking the motion of filaments, and we assume that in the vicinity of a filament, $\Omega$ is much more sharply peaked than $\boldsymbol{u}$ or $\boldsymbol{w}$, we may write

$$
\epsilon \equiv \frac{|\nabla w| /|w|}{\left|\nabla \Omega^{u}\right| /|\Omega|} \ll 1
$$

and note that $\boldsymbol{S}$ is smaller by a factor $\epsilon$ than the convective terms $D \boldsymbol{\Omega}$. This amounts to stating that the filaments should change strength only slowly compared to their advective motion. In this letter, we neglect $S$, so that $\alpha$ remains constant, leaving a more general treatment, including the three-dimensional case, for a later paper.

Let us now suppose we have $N$ total filaments, $N_{u}$ of type $u$ and $N_{w}$ of type $w$, in which the $u$ 's have strength $\pm \alpha_{u}$ and $w$ 's $\pm \alpha_{w}$ (all the same for each species). This system of filaments is Hamiltonian with canonical variables $\left(x_{i},\left|\alpha_{i}\right| y_{i}\right)$. The $\left(x_{i}, y_{i}\right)$ are the normal Cartesian coordinates, taken to be periodic, of the $i$ th filament. The Hamiltonian is

$$
H\left(\boldsymbol{x}_{1}, \ldots, \boldsymbol{x}_{N}\right)=\sum_{i \in u} \sum_{j \in w} \alpha_{i} \alpha_{j} G\left(\boldsymbol{x}_{i} \mid \boldsymbol{x}_{j}\right),
$$

where $G$ is the Green's function for Poisson's equation, $\nabla^{2} G=-\delta\left(\boldsymbol{x}-\boldsymbol{x}^{\prime}\right)$, with appropriate boundary conditions. In an infinite domain, $G\left(\boldsymbol{x} \mid \boldsymbol{x}^{\prime}\right) \propto \ln \left|\boldsymbol{x}-\boldsymbol{x}^{\prime}\right|$. Note also that

$$
\int \boldsymbol{u} \cdot \boldsymbol{w}=\int A^{u} \Omega^{w}=\int A^{w} \Omega^{u}=H .
$$

The Hamiltonian expresses the quantity $\int \boldsymbol{v}^{2}-\boldsymbol{B}^{2}$. There is no self-energy contribution to $H$ because a lone filament has $\boldsymbol{v}$ and $\boldsymbol{B}$ fields of equal magnitude. $H$ plays the role of an 
interaction energy; the sign of the magnetic field energy is reversed because we are neglecting the effects of induction along the convecting filaments. It is perhaps more convenient, however, to view $H$ as a parameter that measures whether the fluid is kinetically or magnetically dominated. Unlike the usual plasma $\beta$, though, which measures the ratio of magnetic energy to microscopic, thermal energy, $H$ measures the difference between the magnetic energy and the kinetic energy due to macroscopic fluid motion. There is a natural boundary at $H=0$. The sign of $H$ can be changed by reversing the signs of the filament strengths of either of the two species. This transformation simply switches the fields $\boldsymbol{v}$ and $\boldsymbol{B}$.

One may determine the equilibrium properties of this system through a calculation like that performed for a guiding-center plasma in Ref. [21]. Taking the micro-canonical ensemble, in which the value of the Hamiltonian is constrained to a constant value $E$, the structure function,

$$
\Phi(E, V, N)=\int \delta(E-H) \prod_{i} d \boldsymbol{x}_{i},
$$

may be explicitly calculated by using the integral representation of the delta function and by invoking a random-phase approximation [22], in which the central limit theorem is applied to obtain the Jacobian for the change of variables from the filament positions $\boldsymbol{x}_{\boldsymbol{i}}$ to the Fourier-transformed filament densities, $\rho_{u}$ and $\rho_{w}$ (with error $O\left(N^{-1}\right)$ ).

The result of this calculation gives

$$
\tilde{\Phi}==\frac{1}{2 \pi} \int d z e^{i z \tilde{E}} \prod_{n_{x}, n_{y}}\left[1+\frac{z^{2}}{\pi^{2} \kappa^{4}}\right]^{-1}
$$

in terms of dimensionless quantities

$$
\tilde{\Phi}=\Phi \frac{\sqrt{N_{u} N_{w}} \alpha_{u} \alpha_{w}}{V^{N}}, \tilde{E}=\frac{E}{\sqrt{N_{u} N_{w}} \alpha_{u} \alpha_{w}},
$$

where $\kappa^{2}=n_{x}^{2}+n_{y}^{2}$ is the dimensionless wavenumber, and $k^{2}=\frac{4 \pi^{2}}{V} \kappa^{2}$. The product in the integrand runs over all $n_{x}, n_{y} \geq 0$ except $n_{x}=n_{y}=0$, and the integral can be reduced to an infinite sum over residues, which occur along le imaginary axis at $z= \pm i \pi \kappa$. One 
immediately sees that $\dot{\Phi}$, unlike the hydrodynamical structure function, is symmetric with respect to $\dot{E}$, and that it differs from a Gaussian distribution in that it asymptotically approaches an exponential function $e^{-\pi \kappa_{\min }|\tilde{E}|}$ as $|\tilde{E}| \rightarrow \pm \infty$. Figure 1 shows $\tilde{\Phi}$, from a numerical evaluation of Eq. (14), along with a Gaussian for comparison, both normalized to 1 . Also shown are histogram data of $\dot{E}$ from an ensemble of vortex systems with fixed filament intensities and random filament positions. The small number of events in the tails of the distribution of $\dot{E}$ causes some scatter in the data, but the data conforms to the calculated structure function in the center and clearly deviates from a Gaussian in the tail. Tokamak experiments have measured deviations from Gaussian probability distributions, which has been taken as an indicator of intermittency in the turbulent edge plasma [23].

One may also calculate the filament density spectra

$$
\begin{aligned}
& \frac{\left\langle\left|\rho_{u}\left(n_{x}^{\prime}, n_{y}^{\prime}\right)\right|^{2}\right\rangle}{N_{u} \alpha_{u}^{2}}=\frac{\left\langle\left|\rho_{w}\left(n_{x}^{\prime}, n_{y}^{\prime}\right)\right|^{2}\right\rangle}{N_{w} \alpha_{w}^{2}} \\
& =\frac{1}{V^{2}} \frac{1}{2 \pi \tilde{\Phi}} \int d z e^{i z \bar{E}}\left[1+\frac{z^{2}}{\pi^{2}\left(n_{x}^{\prime 2}+n_{y}^{\prime 2}\right)^{2}}\right]^{-1} \prod_{n_{x}, n_{y}}\left[1+\frac{z^{2}}{\pi^{2} \kappa^{4}}\right]^{-1},
\end{aligned}
$$

and the cross-correlation spectrum

$$
\begin{aligned}
& \frac{\left\langle\rho_{u}\left(n_{x}^{\prime}, n_{y}^{\prime}\right) \rho_{u}^{*}\left(n_{x}^{\prime}, n_{y}^{\prime}\right)\right\rangle}{\sqrt{N_{u} N_{w}} \alpha_{u} \alpha_{w} V^{2}} \\
& =\frac{1}{2 \pi \tilde{\Phi}} \int d z \frac{-i z}{\pi \kappa^{2}} e^{i z \tilde{E}}\left[1+\frac{z^{2}}{\pi^{2}\left(n_{x}^{\prime 2}+n_{y}^{\prime 2}\right)^{2}}\right]^{-1} \prod_{n_{x}, n_{y}}\left[1+\frac{z^{2}}{\pi^{2} \kappa^{4}}\right]^{-1}
\end{aligned}
$$

Spectra of $\left\langle\left|\rho_{u}\right|^{2}\right\rangle$ and $\left\langle\left|\rho_{w}\right|^{2}\right\rangle$ are even in $E$, while $\left\langle\rho_{u} \rho_{w}^{*}\right\rangle$ is odd in $E$. Both have a pronounced lowest-wavenumber component when $|\tilde{E}| \gg 1$. Ensemble-averaged spectra of vorticity, $\omega \equiv \hat{\boldsymbol{z}} \cdot \boldsymbol{\nabla} \times \boldsymbol{v}$, current, $J \equiv \hat{\boldsymbol{z}} \cdot \boldsymbol{\nabla} \times \boldsymbol{B}$, and cross-helicity, $\boldsymbol{v} \cdot \boldsymbol{B}$, can be calculated by the following relations:

$$
\begin{aligned}
& \left\langle\omega^{2}(\kappa)\right\rangle=\frac{1}{4}\left\langle\left|\rho_{u}(\kappa)+\rho_{w}(\kappa)\right|^{2}\right\rangle, \\
& \left\langle J^{2}(\kappa)\right\rangle=\frac{1}{4}\left\langle\left|\rho_{u}(\kappa)-\rho_{w}(\kappa)\right|^{2}\right\rangle,
\end{aligned}
$$




$$
\langle\boldsymbol{v} \cdot \boldsymbol{B}\rangle=\kappa^{-2} \frac{1}{2}\left(\left\langle\left|\rho_{u}(\kappa)\right|^{2}\right\rangle-\left\langle\left|\rho_{w}(\kappa)\right|^{2}\right\rangle\right)
$$

The spectrum of $\omega^{2}$ always flattens out at large $\kappa$, and when $\tilde{E}$ is large, there is a dominance of vorticity in the longest wavelength mode, such that the longest-wavelength rnode grows proportional to $\check{E}$ as $\check{E} \rightarrow \infty$. As $E \rightarrow-\infty$, the lowest-wavelength mode approaches a constant value $\approx 0.26$. As mentioned before, changing the sign of $E$ interchanges the spectra of $J^{2}$ and $\omega^{2}$, so a concentration of magnetic energy at long wavelengths is expected for $\dot{E} \ll-1$.

Figure 2 shows the spectra of $\omega^{2}$ and $J^{2}$, for $\tilde{E}=2$. The solid and dashed lines are calculated from Eq. (16) and (17). The data points are time-averages from a dynamical simulation of eqs. 8 and 9 using a two-dimensional guiding-center particle-in-cell algorithm [24], modified to account for two species of filaments. The target energy is reached through a random Monty-Carlo procedure, at which point the configuration is saved to become the initial condition of the dynamic simulation. Note that there is some evidence that the trajectories of neutral-fluid vortices are not entirely ergodic [25], although the non-ergodicity appears to be weak [26]. In our case, time-averaged quantities seem to correspond well with the micro-canonical ensemble averages.

The lowest wavenumber mode is dominant for the kinetic energy and suppressed for the magnetic energy. At small $\kappa$, discrepancies are due to random sampling error, but at large $\kappa$, there is a small bias. This could be due to the fact that the code uses a finite size for the particles (while the theory assumes zero diameter), or perhaps represents the failings of the random-phase approximation at large $\kappa$. Figure 3 shows the values of the lowest wavenumber modes, $\omega^{2}\left(k_{\min }\right)$ and $J^{2}\left(k_{\min }\right)$, for positive values of $\tilde{E}$. The asymptotic behaviors $\left\langle\omega^{2}\right\rangle \propto E$ and $\left\langle J^{2}\right\rangle \rightarrow$ const. are clearly demonstrated.

We wish to draw a comparison of these results with those obtained in a series of papers exploring the statistics of two-dimensional MHD turbulence by Fyfe, Joyce, and Montgomery 
[1]. Their analysis used a canonical ensemble based on Fourier modes as phase variables, in the manner of Kraichnan's hydrodynamical turbulence theory [4], in the regime $\langle\boldsymbol{v} \cdot \boldsymbol{B}\rangle=0$, which is obtained in our model when $N_{u} \alpha_{u}^{2}=N_{w} \alpha_{w}^{2}$. Their model predicted the formation of magnetic islands, with the necessary condition $\left\langle\int v^{2}-B^{2}\right\rangle<0$, the same as our condition that $\dot{E}<0$.

The velocity spectra, however, differ significantly. Their analysis always predicts a flat kinetic energy spectrum, even when the magnetic field is identically zero. The reason is that the Fourier statistics are based on the MHD invariants, but as the magnetic field shrinks to zero, the invariants of the system change. The total energy becomes the kinetic energy normally, but the mean squared magnetic potential goes to zero. The enstrophy, meanwhile, changes at a rate proportional to the magnetic field strength. As the magnetic energy vunishes, the enstrophy should be included as an invariant in the calculations. The omission of the enstrophy as an invariant in the analysis dramatically alters the spectrum. The Elsasser variables approach the neutral limit more easily, because none of the field variables vanish for vanishing magnetic field. Instead, the $u$ and $w$ fields approach each other and become identical in the zero magnetic field limit.

Careful account must be made of the invariants of the system's evolution, as failing to account for one of the constants of the motion can completely alter the form of the expected states. In an ideal fluid, there are an infinite number of motion invariants, but most do not survive discrete truncation of the system. In two-dimensional MHD, three invariants which do survive truncation are the total energy, cross-helicity, and mean squared magnetic potential, although no proofs exist which guarantee that these are the only three. Additional headaches arise if one wishes to study MHD in the low magnetic-field limit, in which the magnetic invariants approach zero identically, but the enstrophy begins to change slowly enough that it should also be counted as an invariant. Throughout, of course, careful attention must be paid to time scales. If one waits long enough, any non-zero magnetic field 
will destroy the constancy of the enstrophy, while at the same time, viscous and ohmic dissipation whittle away at the inviscid invariants. Eventually, we expect that the effects of neglecting the sources in equation Eq. (5) will also make themselves felt.

This work was supported by the National Science Foundation and the U.S. Department of Energy contract \#DE-FG05-80ET-53088. 


\section{References}

[1] D. Fyfe, D. Montgomery, \& G. Joyce, J. Plasma Physics 17, 369 (1977), and references therein.

[2] J.B. Taylor \& B. McNamara, Phys. Fluids 14, 1492 (1971).

[3] A. Royer, J. Math. Phys. 25. 2873 (1984).

[4] R.H. Kraichnan \& D. Montgomery, Rep. Prog. Phys. 45, 547 (1980).

[5] J.C. McWilliams, J. Fluid Mech. 146, 21 (1984).

[6] R. Robert \& J. Sommeria, J. Fluid Mech. 229, 291 (1991).

[7] J. Miller, Phys. Rev. Lett. 64, 2137 (1990).

[8] J.M. Kosterlitz, J. Phys. C, 7, 1046 (1974).

[9] T.S. Lundgren and Y.B. Pointin, Phys. Fluids 20, 356 (1977).

[10] R.A. Simith, Phys. Rev. A 43, 1126 (1991).

[11] V. Berdichersky, I. Kunin, and F. Hussain, Phys. Rev. A 432050 (1991).

[12] L.J. Campbell and K. O'Neil, J. Stat. Phys. 65, 495 (1991).

[13] W.H. Matthaeus, W.T. Stribling, D. Martinez, S. Oughton, and D. Montgomery, Physica D 51, 531 (1991);

D. Montgomery, W.H. Matthaeus, W.T. Stribling, D. Martinez, and S. Oughton, Phys. Fluids A 4. 3 (1992). 
[14] G.F. Carnevale, J.C. McWilliams, Y. Pomeau, J.B. Weiss, and W.R. Young, Phys. Fluids A 4, 1314 (1992).

[15] G.F. Carnevale, J.C. McWilliams, Y. Pomeau, J.B. Weiss, \& W.R. Young, Phys. Rev. Lett. 662735 (1991);

J.B. Weiss \& J.C. McWilliams, Phys. Fluids A, in press.

[16] S. Kida, J. Fluid Mech., 112, 397 (1981).

[17] A. Pumir and E. Siggia, Phys. Fluids 30 (6), 1606 (1987).

[18] F. Yusef-Zadeh, in The Center of the Galaxy (Boston: Kluwer, 1988), p. 243; Physics of Magnetic Flux Ropes, ed. C.'T. Russell, E.R. Priest, and L.C. Lee (Washington D.C.: AGU, 1990);

H. Zirin, Astrophysics of the Sun (C'ambridge: Cambridge University Press, 1988).

[19] D. Biskamp and H. Welter, Phys. Fluids B 2, 1787 (1990), and references therein.

[20] W.M. Elsasser, Phys. Rev. 79, 183 (1950).

[21] S.F. Edwards \& J.B. Taylor, Proc. R. Soc. Lond. 336A, 257 (1974).

[22] S.E. Seyler, Jr. Phys. Fluids 19 1336, (1976).

[23] R. Jha, P.K. Kaw, S.K. Mattoo, et al, Phys. Rev. Lett. 69, 1375 (1992).

[24] W.W. Lee \& H. Okuda, J. Comp. Phys. 26, 1.39 (1978).

[25] K.M. Khanin, Physica D 4, 261 (1982).

[26] J.B. Weiss \& J.C. McWilliams, Phys. Fluids A 3835 (1991). 


\section{Figure Captions}

Fig. 1

Structure function $\tilde{\Phi}$, showing phase-space probability density as a function of , >. the energy $\tilde{E}$. Solid line is computed from Eq. (14), and data points are measured histogram frequencies from a set of random configurations. The dotted line is a normalized Gaussian chosen to match at $\tilde{E}=0$, shown for comparison.

Fig. 2

$\left\langle J^{2}\right\rangle$ and $\left\langle\omega^{2}\right\rangle$ spectra for $\check{E}=2$. Data poinis are time-averaged from a dynamical simulation. Solid and dashed lines are spectra predicted from the micro-canonical ensemble.

Fig. 3

Values of the lowest-wavenumber mode vs. $\tilde{E}$ from dynamical runs and theoretical predictions. 
Fig. 1

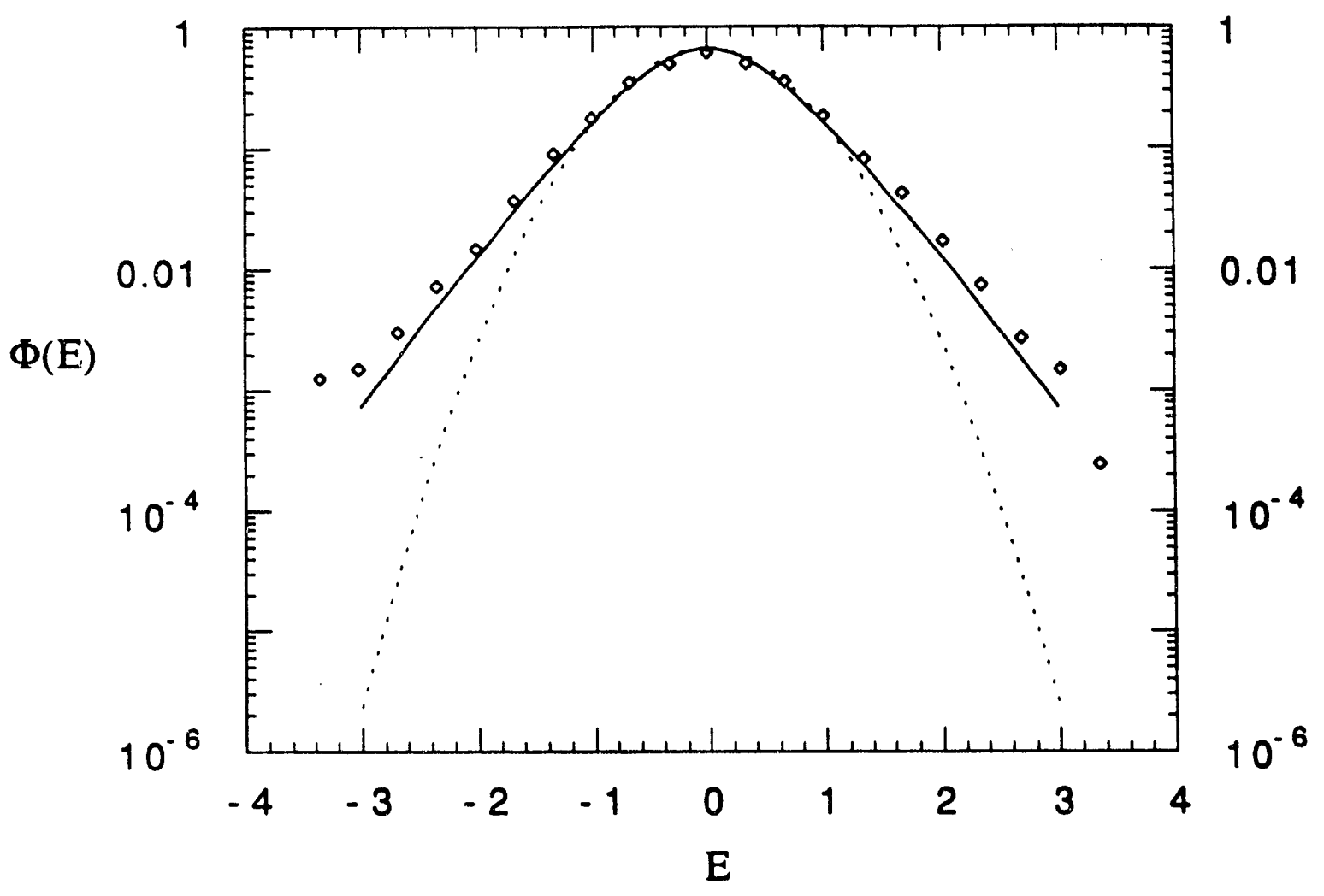


Fig. 2

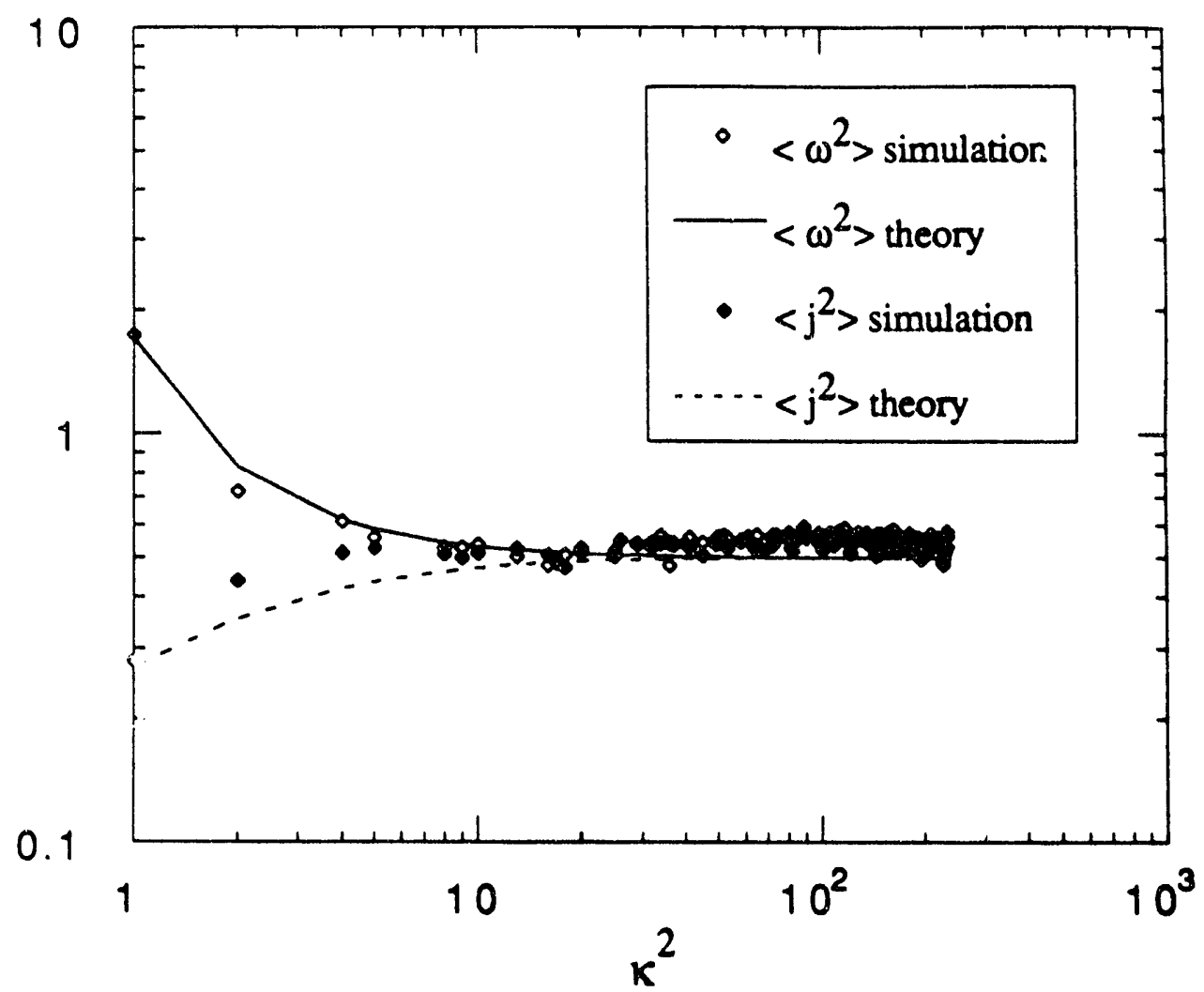


Fig. 3

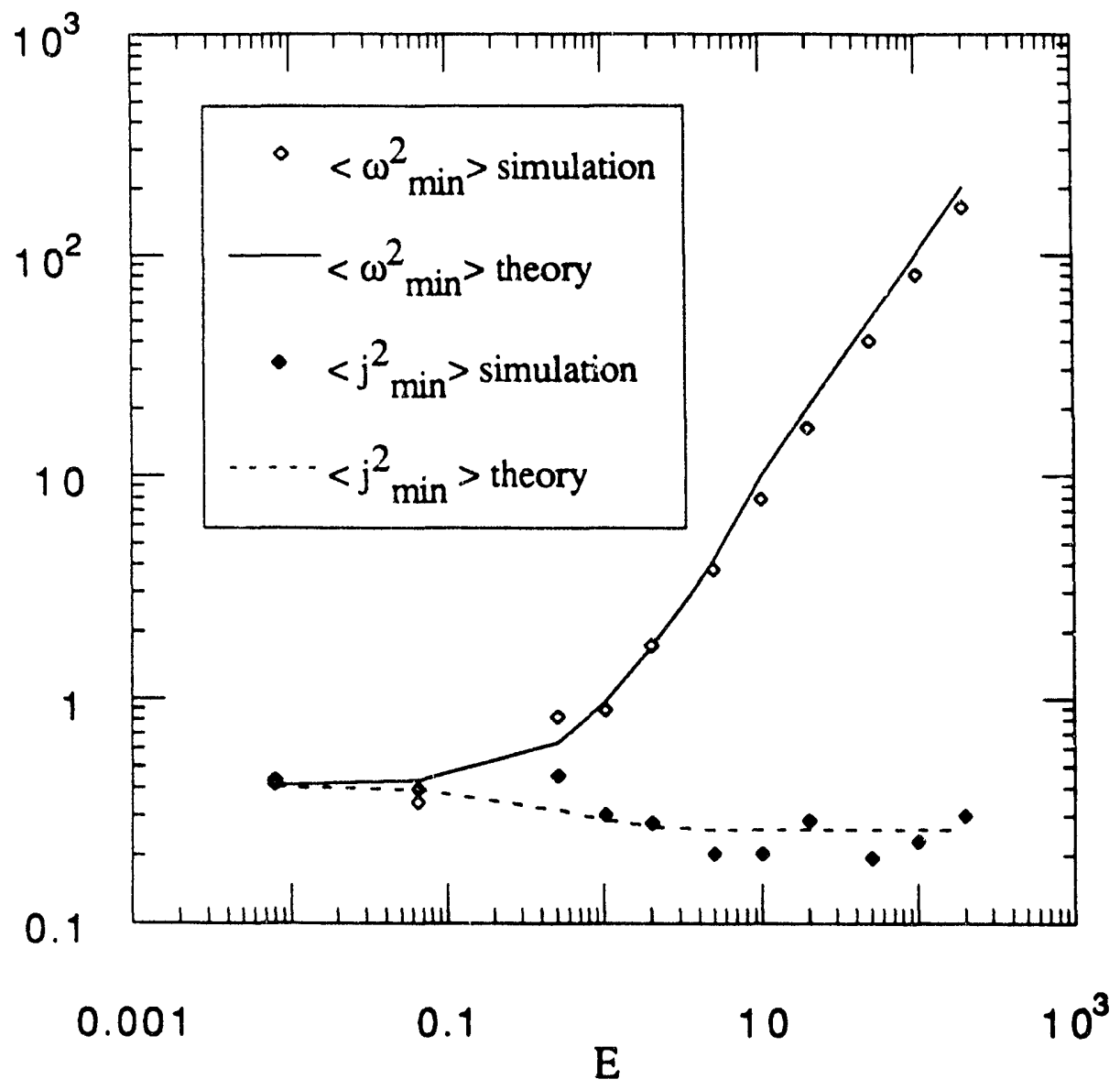



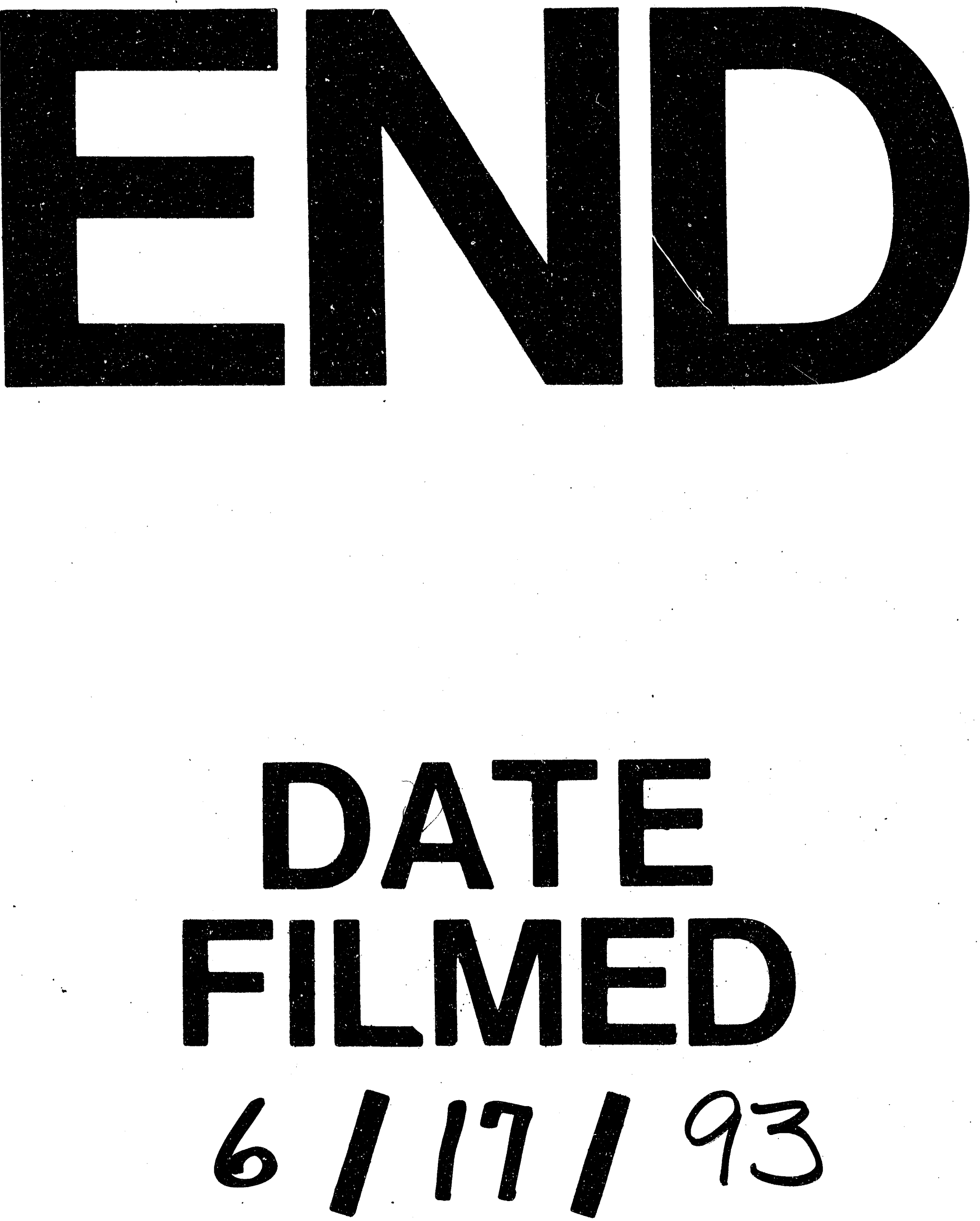
